SLAC-PUB-7100

March 1996

\section{ARE PARTONS CONFINED TACHYONS? ${ }^{\star}$}

\author{
H. Pierre Noyes
}

Stanford Linear Accelerator Center

Stanford University, Stanford, California 94309

\section{ABSTRACT}

We note that if hadrons are gravitationally stabilized "black holes", as discrete physics suggests, it is possible that PARTONS, and in particular quarks, could be modeled as tachyons, i.e. particles having $v^{2}>c^{2}$, without conflict with the observational fact that neither quarks nor tachyons have appeared as "free particles". Some consequences of this model are explored.

\section{MASTER}

Invited paper presented at the

Twelfth Annual International Meeting of the Western Chapter of the ALTERNATIVE NATURAL PHILOSOPHY ASSOCIATION

Cordura Hall, Stanford University, February 17-19, 1996

* Work supported by the Department of Energy, contract DE-AC03-76SF00515.

\section{INTRODUCTION}

Kendall, Friedman and Taylor received the Nobel prize for their discovery of hard scattering centers inside the proton when it is bombarded with high energy electrons. These centers are reminiscent of the nuclei discovered by Rutherford when he bombarded atoms with $\alpha$-particles. Unfortunately for simplicity they do not have as transparent a geometrical interpretation. These "deep inelastic" electron scattering experiments were analyzed by Bjorken and shown to follow the relativistic kinematics of scattering from electrically charged objects "within" the proton, so the analogy to Rutherford scattering is indeed close, once one recognizes that, up to a point, the enormously different energy scale makes little difference. Feynman arrived at similar conclusions and dubbed these scattering centers "partons", but was careful to point out that all the essential features of his own work were already present in Bjorken's analysis.

It was soon recognized that these scattering centers might indeed be the "quarks" postulated by Gell-Mann or the "Aces, Deuces and Treys" of Zweig. These theoretical entities had been invented to account for a number of regularities in the hadronic mass spectrum and strong interaction dynamics of elementary particles. So the deep inelastic scattering analysis soon came to be called the "quark-parton" model. "Free quarks" were eagerly sought in a number of ingenious ways, but so far have made themselves conspicuous by their absence. This embarrassment was eventually removed by the quantum-chromodynamic strand of what is now called the "standard model of quarks and leptons". In contrast to leptons whose "running" electromagnetic (and, in principle, weak) coupling constants become ever stronger as the energy of the bombarding particles increases, the quantum chromodynamic or strong coupling constant becomes ever weaker as the energy increases leading to what is called "asymptotic freedom". But the same model implies that the quark coupling constant becomes ever stronger as the energy becomes smaller leading to "infrared slavery" and quark confinement within the hadrons. Unfortunately making an actual calculation of the "confining potential" from first principles has as yet proved to lie beyond the ingenuity of theorists; direct attack on the problem using super-computers is only now beginning to yield believable - and not very accurate - results. Here we use some clues from our new fundamental theory ${ }^{[1,2]}$ to construct a different dynamical model for quark confinement which might be easier to compute.

That quarks are confined in our theory was argued some time ago. According to McGoveran's Theorem, ${ }^{[3,4]}$ in any finite and discrete theory with a universal ordering operator there can be no more than 3 homogeneous and isotropic macroscopic ("spacial") dimensions. These are exhausted in labeling particles by the three absolutely conserved quantum numbers allowed in current particle physics. 
There are four candidates: lepton number, baryon number, charge and either the "z-component of weak isospin" or "weak hypercharge". The extended GellMannNishijima relation leaves only three of these to specify independently and measure at macroscopic distances. Any other conserved, discrete quantum number must "compactify" or be "confined". Since quarks and gluons carry the conserved quantum number of color, we conclude that this quantum number cannot appear as a label for free particles and hence that gluons and quarks are confined. But this general argument does not lead to a computational scheme.

There is another theoretical entity which has never been observed, namely any massive particle traveling faster than the speed of light. The generic name for such particles is "tachyon", perhaps more familiar to some of my readers as part of the technobable in the STAR TREK series than in the scientific literature. The possibility of their existence was explored extensively by Gary Feinberg, ${ }^{[5]}$ and others. ${ }^{[6,7]}$ There is no direct experimental evidence that such particles can be made to appear in the laboratory. Since neither quarks nor tachyons have been observed directly we adopt here the speculative stance that both might turn out to be the same type of entity, and try to construct a model that achieves this in a natural way. In Newtonian physics, if an attracting center is massive enough so that circular velocity is $c$ at some radius $R$, neither light nor particles limited to that velocity at $\mathrm{R}$ can ever escape this "black hole", as was first pointed out by Laplace in 1795. In the Newtonian framework, particles in elliptical orbits tangent to this confining circle at aphelion would necessarily have still larger velocities as they plunged toward and swung around the attracting center, and hence could be called "confined tachyons". This, in short, is our fanciful dynamical parton-tachyon model.

Conventional physicists will reject this idea out of hand because I have taken a Newtonian picture and extended it into the supraluminal region where it is wildly inappropriate. If I ended my remarks here, I would have to agree with them. But in discrete physics we have found that apparently "non-relativistic" ideas can find strange connections with relativistic physics, for instance in the successful derivation of Maxwell's Equations from superficially "non-relativistic" commutation relations and Newton's second law of motion. ${ }^{[8]}$ So I am prepared to pursue this wild idea here and see what comes of it.

\section{THE CLASSICAL MODEL}

We start by assuming that we know nothing about relativity and quantum mechanics other than that the velocity of light $c$ has a special significance in both theories. Then, following Laplace, we note that there are can be interactions (in his case, gravitational) strong enough so that particles with velocity $c$ cannot escape the neighborhood of the source of this interaction. Our approach is kinematic, rather than dynamic, in that we take as paradigmatic a conic section trajectory. For trajectories obeying Kepler's Laws, it can be shown that these (a) conserve specific angular momentum or angular momentum per unit mass about the attracting center given by $r v_{\perp}=r^{2} \dot{\theta}$ where $r$ is the distance from that center, $v_{\perp}$ is the component of velocity perpendicular to the line from the center to the point on the trajectory, and $\theta$ is the angle that line (in the plane of the trajectory) makes with a line from the center to the perihelion point and (b) that the acceleration of the point is toward the center and varies inversely with the square of the distance. These kinematic statements are derived from Kepler's Laws in the Appendix.

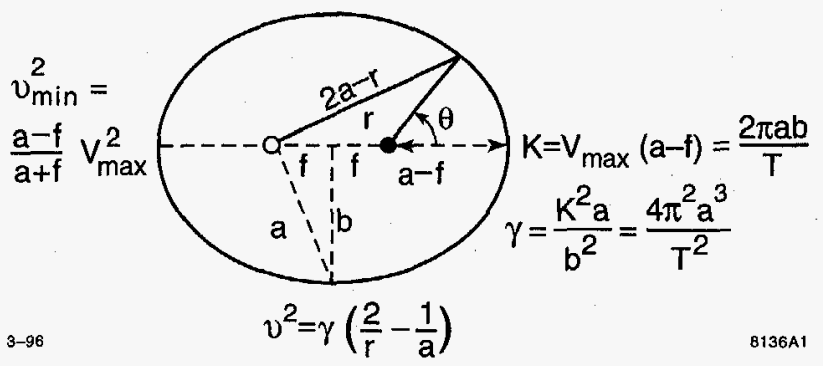

Figure 1. Radial coordinates and algebraic parameters for elliptical motion.

This derivation also provides us with the generalized equation for conic section motion with velocity $v$ along such a trajectory whose square is $v^{2}=\dot{r}^{2}+(r \dot{\theta})^{2}$

$$
\begin{aligned}
& v^{2}=\gamma\left(\frac{2}{r}-\frac{1}{a}\right) \text { elliptical } \\
& v^{2}=\gamma\left(\frac{2}{r}\right) \quad \text { parabolic } \\
& v^{2}=\gamma\left(\frac{2}{r}+\frac{1}{a}\right) \text { hyperbolic }
\end{aligned}
$$

We illustrate the radial coordinates for elliptical motion in Fig. 1 and for hyperbolic motion in Fig. 2. To calculate the constant $\gamma$ from space-time measurements on 


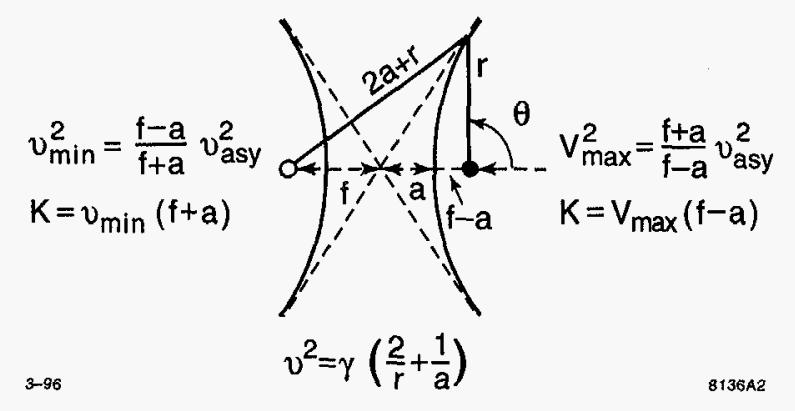

Figure 2. Radial coordinates and algebraic parameters for hyperbolic motion.

elliptical orbits rather than dynamically (i.e. by first introducing the concepts of force and inertial mass), we symbolize the specific angular momentum by $K \equiv$ $r v_{\perp}=r^{2} \dot{\theta}$ and use Kepler's Second and Third Laws (cf. Appendix) to obtain the relations

$$
\gamma=\frac{K^{2} a}{b^{2}}=\frac{4 \pi^{2} a^{3}}{T^{2}}
$$

where $T$ is the period, that is the time it takes a point moving on an elliptical trajectory to return to the same position. If the acceleration is centrifugal (away from the center) we call it repulsive rather than attractive. Such motion is necessarily hyperbolic, and motion takes place on the opposite branch of the hyperbola (cf. Fig. 2). For this case (eg Rutherford scattering), the trajectory equation is given by

$$
\frac{1}{r}=-\frac{a+f \cos \theta}{f^{2}-a^{2}}
$$

but the equation for the square of the velocity remains unchanged.

These results, being classical, are all scale invariant. We now analyze how separate them into two types of motion, in the first of which (particulate motion), the velocity is always less than $c$, and the second of which (confined tachyonic motion) always has velocities greater than $c$. To do this we first note that, for attractive interactions, the velocity is always maximum at the distance of closest approach to the attracting center $r_{\min }=|a-f|$. If we have velocity close to but below $c$ at this point, any case of particulate motion will, necessarily, lie outside the circle of radius

$$
c^{2} r_{\min }^{(c)}=\gamma_{c}
$$

For elliptical $(a>f)$ motion close to the limiting case, the velocity will fall to $\frac{a-f}{a+f} c$ at $r_{\max }$ (apehelion). For hyperbolic motion $(f>a)$, the velocity will fall to $\frac{f-a}{f+a} c$ along the asymptote; the particle escapes. At the distance of closest approach the motion is always perpendicular to the line from the object to the center, so in all cases, the specific angular momentum is $K_{c}=r_{\min } c=|a-f| c$. If the distance of closest approach $r_{\min }^{(v)}$ is greater than $r_{m i n}^{(c)}$, then the velocity $v_{\max }$ is always less than $c$, and we have that

$$
\frac{|a-f|}{a+f} v_{m a x}^{(v)} \leq v \leq v_{m a x}^{(v)}
$$

where the lower limit on the velocity is either the apehelion velocity for elliptical motion, or the asymptotic velocity for hyperbolic motion. Clearly the specific angular momentum is always given by $K_{v}=|a-f| v_{\max }$

If the maximum distance from the attractive center is less than $r_{m i n}^{(c)}$, the trajectory is always elliptical and always tachyonic.

For repulsive interactions the asymptotic velocity is the maximum velocity and is always less than $c$, the motion is always hyperbolic and the velocity falls to its minimum value at the distance of closest approach, which is $a+f$. Clearly for repulsive interactions $K_{v}=(a+f) v_{m i n}$ and is zero for the straight line trajectory where the asymptotic velocity is pointed straight at the center.

We trust the classical kinematics of our confined tachyon motion inside the "black hole radius" $r_{\min }^{(c)}$ and the allowed particulate motion outside with $v<c$ is now clear, and that for a given interaction strength $\gamma_{c}$ the two regions are cleanly separated by that radius. Before showing how the two regions can communicate, and why we have excluded the (classically allowed) cases of an object with $v<c$ at large distances but $v>c$ at short distances, we must first examine the modifications that relativistic quantum mechanics imposes on our classical model.

\section{THE QUANTUM MODEL}

Our quantum model is basically that discussed in the paper on the fine structure spectrum of hydrogen ${ }^{[9]}$ in which David McGoveran and I showed that the Sommerfeld formula and an improvement in accuracy by four orders of magnitude for the calculation of the fine structure constant could be obtained combinatorially. There we derived the formula

$$
\left(\frac{E_{n}}{m_{e p} c^{2}}\right)^{2}\left[1+\left(\frac{1}{137 n}\right)^{2}\right]=1
$$

where $m_{e p}=m_{e} m_{p} /\left(m_{e}+m_{p}\right), m_{e}, m_{p}$ are the masses of the electron and proton respectively, and $E_{n}=\left(m_{p}+m_{e}\right) c^{2}-\epsilon_{n}$ is the mass-energy of their $n^{\text {th }}$ bound state, 
$\epsilon_{n}$ is the binding energy of that state, $n$ is Bohr's principle quantum number for hydrogen and $1 / 137$ is the combinatorial calculation of the fine structure constant $e^{2} / \hbar c$. This formula (using empirical values) is equivalent to Bohr's relativistic correction to his non-relativistic model for the hydrogen atom, and was published by him in $1915^{[10\}}$

Using the geometrical-kinematic approach we employed above, we could model a quantized circular orbit for the same problem ${ }^{[1]}$ as a regular polygon with $n$ sides of length $137 n h / m_{e} c$. Each side is traversed with velocity $c / 137 n$, with the direction but not the magnitude changing at each corner of the polygon. This model leads to the same formula for the energy and period of the motion.

In the fine structure paper we also point out that the general formula for a (relativistic) bound state of mass $\mu$ composed of exactly two particles with masses $m_{1}, m_{2}$ due to an interaction measured by the coupling constraint $g^{2} / \hbar c=1 / 137 n$ in a S-matrix theory give the equivalent formula (with $\hbar=1=c$ )

$$
\left(g_{\mu m_{1} m_{2}}^{2} \mu\right)^{2}=\left(m_{1}+m_{2}\right)^{2}-\mu^{2}
$$

We also pointed out that this formula is non-perturbative and gives a good value for the basic (phenomenological) strong interaction coupling constant $G_{\pi N}^{2} \bar{N}$ between pions and nucleons.

In a subsequent paper ${ }^{[12]}$ we noted that the same formula could also be derived by assuming that the relativistic energy allowed to a "vacuum fluctuation" in a finite and discrete "second quantized field theory" which has at most $N$ particles of mass $m$ is $M_{x}=N m$. Then a bound state of mass $\mu$ "at rest" can virtually dissociate into a system with that energy balanced by the momenta of $N$ quanta of momentum $\mu c$. This allows us to write that

$$
\mu^{2}=(N m)^{2}-(N \mu)^{2}=E^{2}-p^{2}
$$

The handy-dandy formula (3.2) is recovered by taking $g^{2}=1 / N$ and $m=m_{1}+m_{2}$. We conclude that this is formula is the proper starting point for the relativistic quantum mechanical connection between the coupling constant between a particle of mass $\mu$ and its two constituents and the mass values themselves.

We have seen that "classical" tachyons are obtained by replacing the invariant relation $E^{2}-p^{2}=m^{2}$ by the relation $p^{2}-E^{2}=\mu^{2}$ thus guaranteeing that $v=p / E \geq 1$ in units of the limiting velocity $c$. It thus seems that, so far as the two-body problem goes, the handy-dandy formula, and hence the relativistic Rutherford formula, should hold just as well for confined tachyons as for confined quarks. This immediately explains why the parton Bohr formula (and the BohrSommerfeld generalization) proved so immediately useful as a guide to the discover and exploration of the hydrogen-like energy levels of "quarkonium", i.e. the bound states of a quark-antiquark pair.

This is fully compatible with the derivation of the handy-dandy formula from the S-matrix bound state wave function normalization condition $\int \phi=1$ where $\phi(s)=\frac{g^{2} \mu}{s-\mu^{2}}$ and $s$ is square of the invariant four momentum. The form follows from the definition of a bound state as a pole in the S-matrix, and gives us explicitly

$$
\int_{\left(m_{1}+m_{2}\right)^{2}}^{\infty} \phi^{2}(s) d s=1
$$

leading to the handy dandy formula, Eq.3.2. In the usual interpretation the "off mass shell" values of run from the rest-energy (momentum equal to zero) value of $\left(m_{1}+m_{2}\right) c^{2}$ to infinity. But for tachyons, the momentum runs from the minimum value $\left(m_{1}+m_{2}\right) c$ (energy equal to zero) to infinity. The algebraic consequence is the same!

\section{Conclusion}

We conclude that, carefully interpreted, from an S-matrix point of view, once one accepts that quarks, or partons, or tachyons are confined, the paradigmatic aspects of the quark-parton model can be extracted just as well from a confined tachyon model. It then becomes a question of which is most useful as an aid to the imagination in formulating the interactions. For some who like to think classically, tachyons orbiting inside a black hole may seem a better place to start than either quantum field theory or discrete physics. This little exercise is offered to them as a starting point which, like discrete physics but unlike quantum field theory, has a well defined "correspondence limit", even though that limit has the usual problem of any black hole model of whether or not information can be successfully extracted once particles have passed the event horizon. 


\section{Appendix: KEPLER'S LAWS AND INVERSE SQUARE LAW ACCELERATIONS}

\subsection{KEPLER,S LAWS}

Since my starting point for discussing tachyons is a kinematic rather than a dynamical model for confined motion in circular and elliptical orbits, I give here a derivation of this model from Kepler's Laws taken from draft chapter of my book on discrete physics. ${ }^{[13]}$ I state Kepler's Laws as follows:

I. The paths of the planets are ellipses with the sun at one, single focus

II. The line from sun to planet sweeps out equal areas in equal times.

III. The ratio of the square of the periodic time for the planet to complete its orbit to the cube of the length of the major axis is independent of the length of the minor axis and the same for all planets.

Starting with the First Law, we draw the ellipse (cf. Fig. 1) with the line between the sun and the planet of length $r$, and $\theta$ the angle between this line and the line from the sun to the perihelion point (distance of closest approach to the sun). Since an ellipse is defined to be the locus of all points such that the lengths of the lines from the planet to the two foci add to some constant value which we call $2 a$, if the distance between the two foci is called $2 f$, the two foci and the planet define a triangle with sides $r, 2 f, 2 a-r$. Using the fact that for any triangle with sides $a, b, c$, we know that $2 a b \cos \theta_{a b}=a^{2}+b^{2}-c^{2}$, we have that

$$
2 r f \cos (\pi-\theta)=r^{2}+4 f^{2}-(2 a-r)^{2}
$$

from which follows the orbit equation connecting $r$ and $\theta$, namely

$$
\frac{1}{r}=\frac{a+f \cos \theta}{a^{2}-f^{2}}=\frac{a+f \cos \theta}{b^{2}}
$$

Here $a$ is called the semi-major axis of the ellipse. As we can see from Fig. 1 perihelion distance is $a-f$, aphelion distance is $a+f$ and the square of the semi-minor axis, or half-width, of the ellipse is given by $b^{2}=a^{2}-f^{2}$.

Turning to the Second Law, we recall that for a triangle with sides $a, b, c$ the square of the area is given by $A^{2}=\frac{1}{16}\left[(a+b)^{2}-c^{2}\right]\left[c^{2}-(a-b)^{2}\right]$. Hence the area swept out when we go from position $r$ to $r+\Delta r$ due to velocity $v$ in time $\Delta t$ is given by

$$
\Delta A^{2}=\frac{1}{16}\left[(2 r+\Delta r)^{2}-v^{2} \Delta t^{2}\right]\left[v^{2} \Delta t^{2}-\Delta r^{2}\right]
$$

We first note that the velocity component along $\mathrm{r}$ is $\dot{r}=\frac{\Delta r}{\Delta t}$ and that the square of the velocity component perpendicular to $\mathrm{r}, v_{\perp}=r \dot{\theta}$, is

$$
v_{\perp}^{2}=v^{2}-\dot{r}^{2}=r^{2} \dot{\theta}^{2}
$$

Hence, to the extent that we can neglect $\Delta r$ and $v \Delta t$ compared to $2 r$, we have that

$$
\frac{\Delta A}{\Delta t}=\frac{1}{2} r v_{\perp}=\frac{1}{2} r^{2} \dot{\theta}
$$

In Newtonian dynamics the quantity $m r v_{\perp}=m r^{2} \dot{\theta}$ is called the angular momentum (of a particle of mass $m$ moving past a point at a distance $r$ from it). Unfortunately there is no accepted term for the quantity $r v_{\perp}$. We choose to call it specific angular momentum and note that $r v_{\perp}=r^{2} \dot{\theta}$. It has dimensions of angular momentum per unit mass, or $\mathbf{L}^{2} / \mathbf{T}$, or area per unit time. When it is conserved in particle motion, we will call it Kepler's Constant and symbolize it by $K$. If the planet takes a time $T$ (its period) to return to the same position in its orbit, the line from sun to planet sweeps out the area of the ellipse, which is $\pi a b$. Hence

$$
K=r v_{\perp}=r^{2} \dot{\theta}=\frac{2 \pi a b}{T}
$$

independent of time

In order to relate this motion to Kepler's Third Law, namely that $a^{3} / T^{2}$ has the same value for all planets, we first differentiate the orbit equation to obtain a connection between the square of the velocity, the radial position, Kepler's constant and the parameters of the ellipse. Since

$$
\dot{r}=\frac{f \sin \theta r^{2} \dot{\theta}}{b^{2}}=\frac{f \sin \theta}{b^{2}} K ; \quad r \dot{\theta}=\frac{K}{r}
$$

we have that

$$
v^{2}=K^{2}\left[\frac{f^{2} \sin ^{2} \theta}{b^{4}}+\frac{1}{r^{2}}\right]=\frac{K^{2} a}{b^{2}}\left(\frac{2}{r}-\frac{1}{a}\right)=\gamma\left(\frac{2}{r}-\frac{1}{a}\right)
$$

where we have used Kepler's Third Law to define the constant

$$
\gamma=\frac{K^{2} a}{b^{2}}=\frac{4 \pi^{2} a^{3}}{T^{2}}
$$

This shows that our velocity equation applies to any planet in the solar system. Note that it is independent of the focal distance $f$ or the eccentricity of the orbit $\epsilon=f / a$ or the semi-major axis $b^{2}=a^{2}-f^{2}=a^{2}\left(1-\epsilon^{2}\right)$. The velocity equation 
for a circular orbit $(r=a=b)$ defines a particular specific angular momentum, $K_{a}$, and velocity $V_{a}$ which relate to the orbital parameters in the following way

$$
V_{a}=\frac{K_{a}}{a}=\frac{2 \pi a}{T}=\frac{\gamma}{K_{a}}
$$

and allow us to bound the velocities in orbit as follows:

$$
\frac{a-f}{a+f} \leq \frac{v^{2}}{V_{a}^{2}} \leq \frac{a+f}{a-f}
$$

The velocity equation, which is the key to solar system dynamics and the technological requirements for interplanetary travel, ${ }^{[14]}$ is often derived using the conservation of energy, and other concepts taken from Newtonian and post-Newtonian physics. We emphasize that the derivation given here is purely kinematic, given Kepler's three laws.

\subsection{THE INVERSE SQUARE LAW}

Our next step, drawing on Newton's analysis of these laws, is to emphasize is the key role played by the concept of change in velocity or acceleration. For the planetary problem, we start by differentiating the equation for radial velocity in an orbit to obtain

$$
\ddot{r}=\frac{f \cos \theta \dot{\theta} K}{b^{2}}=\left(\frac{1}{r}-\frac{a}{b^{2}}\right) \frac{K^{2}}{r^{2}}=\frac{K^{2}}{r^{3}}-\frac{\gamma}{r^{2}}
$$

The first term, called the centrifugal acceleration, arises because we have constrained the motion to move in an orbit obeying Kepler's Second Law and depends (through $K$ ) on the orbital parameters $a, b, T$ for the individual planet. But the second applies to all the planets in the solar system, and varies with the inverse square of the distance from sun to planet.

One of Newton's great insights came when he realized that the motion of the Galilean satellites about Jupiter also obeyed Kepler's laws with Jupiter playing the role of the sun and that our own satellite (the moon) could be thought of as falling around the earth. This suggests that for each gravitating center there is some constant $\gamma$ for centripetal acceleration toward that center given by $\gamma / r^{2}$. In the solar system, Newton could identify $\gamma_{S u n}$ common to all the planets, $\gamma_{J u p i t e r}$ for Galilean satellites relative to Jupiter and $\gamma_{E a r t h}$ for the motion of our own moon relative to the Earth. But then, for an object with $K=0$ at the surface of the earth, $r=R_{E}$, the acceleration toward the center of the earth could be identified with the post-Galilean physical constant $g$ used to describe the motion of freely falling objects close to the surface of the earth by taking

$$
g=\frac{\gamma_{E}}{R_{E}^{2}}=\frac{4 \pi^{2} a_{M}^{3}}{T_{M}^{2} R_{E}^{2}}
$$

Note that the radius of the earth, $R_{E}$ had been measured by Erosthenes in terms of local earth distances long ago, the length of the month, $T_{M}$, was known in seconds, and the semi-major axis of the moon's orbit, $a_{M}$ was measured in terms of the earth's radius by the parallax of the moon sighted from well separated positions on the earth at the same time. Therefore the value of $g$ could be calculated from known quantities and compared with local measurements of $g$ in the same units of length and time.

Here I abandon this (hopefully well known) pseudo-historical account to emphasize that the mathematical analysis we have carried through will apply to any geometrically describable motion which follows elliptical (and more generally, conic section) paths conserving specific angular momentum (Kepler's Second Law), and ties these firmly to inverse square law accelerations. Thus this analysis applies to the motion of charges which obey Coulomb's Law (a fact which Rutherford exploited to arrive at his model of the nuclear atom), and could be applied to a quark model mediated by massless gluons if we can make sense out of such a bizzare "classical" model for strong interactions. Newton, of course, went on from his analysis to postulate the law of universal gravitation, which for the restricted case we have treated of a test object of mass small compared to the attracting center of mass $M$ amounts to asserting that the constant $\gamma$ we isolated above has the value $G M$ where $G$ is a universal (but in Newton's time unmeasurable) constant. Thus only relative planetary masses were known until Cavendish made a terrestrial measurement of $G$ and hence was said to have "weighed the earth" (and the sun!). Coulomb's measurements provided (in principle) an absolute value for charges in an analagous way, but we will have to do a lot more work before our model can give an absolute measurement of the basic strong interaction.

\subsection{THE VELOCITY OF Light}

One of Galileo's many inventions was a method of determining longitude by observing the local time at which a satellite of Jupiter was eclipsed by Jupiter, and comparing this with the time the same exclipse could be observed at some standard location. He did not publish it because he was negotiating with Spain to pay him for developing the necessary tables of eclipses, and the negotiations did not succeed before he was distracted by other events. A century later determination 
of longitude was still a matter of great practical importance. Quantitative investigation of the variation of eclipse times compared to those predicted by Kepler's Laws eventually revealed a puzzling discrepancy periodic in the synodic period $1 / T_{E J}=\left(1 / T_{E}-1 / T_{J}\right)$ with which the distance between Earth and Jupiter varies. In $1727 \mathrm{R} \phi$ mer noticed that if the light coming from Jupiter to Earth has a finite velocity, then this could quantitatively account for the discrepancy and in so doing provide a measurement of the velocity of light, now usually symbolized by $c$. His value is in fact within $30 \%$ of more recent determinations. As we now know, this physical constant has a much more general significance than this, and is now recognized as a universal constant on a par with $G$.

In 1795 Laplace noted that this has a peculiar implication. ${ }^{[15]}$ We have given above the velocity equation for any material object subject to gravity; it was extended by Newton to include the parabolic and hyperbolic conic sections as well:

$$
\begin{aligned}
& v^{2}=V_{a}^{2}\left(\frac{2}{r}-\frac{1}{a}\right) \text { elliptical } \\
& v^{2}=V_{a}^{2}\left(\frac{2}{r}\right) \quad \text { parabolic } \\
& v^{2}=V_{a}^{2}\left(\frac{2}{r}+\frac{1}{a}\right) \text { hyperbolic }
\end{aligned}
$$

Hence any object subject to gravity which has a squared velocity at perihelion greater than $2 V_{a}^{2}$ can escape on a hyperbolic trajectory, but if it has less, it will remain trapped in orbit, or (if less than $V_{a}^{2}$ ) never even achieve orbit. Laplace assumed that light acts in this way, and calculated that a massive object with the density of the earth and 250 times the radius of the sun would have so strong a surface gravity that light could never leave its surface. We now have much stronger reasons than Laplace to believe that light is subject to gravity, and some observational evidence for "black holes" which cannot (of course) be seen but which cause what can be interpreted as gravitational phenomena that are visible at sufficient distances from their centers.

Using our Newtonian formulae for the case of $V_{a}^{2}=c^{2}$, the radius of such an object is related to its mass by

$$
R_{b h}=\frac{G M_{b h}}{c^{2}}
$$

We see that neither $G$ nor $c$ by themselves break classical scale invariance, but if we can find some natural phenomenon that sets the scale of masses, or lengths, or times, or angular momenta, we will have ready to hand a way to set a scale which takes us from familiar "classical" laws of physics into a realm of new phenomena where those laws may not apply.

\section{REFERENCES}

1. H. P. Noyes, "Some Remarks on Discrete Physics as an Ultimate Dynamical Theory", Proc. ANPA 17 (in press) and SLAC-PUB-95-7017, Oct. 1995.

2. H. P. Noyes, "A Bit-String 'Theory of Everything'", in Proc. Workshop on Physics and Computation (PhysComp '94), D. Matzke, ch., IEEE Comp. Soc. Press, Los Amitos, CA, 1994, pp. 88-94.

3. D. O. McGoveran and H. P. Noyes, "Foundations of a Discrete Physics" in Discrete and Combinatorial Physics (Proc. ANPA 9), H. P. Noyes, ed, ANPA WEST, 112 Blackburn Ave., Menlo Park, CA 94025, 1987, theorem 13, p.59; and SLAC-PUB-4526 (June, 1989), p.32.

4. H. P. Noyes and D. O. McGoveran, Physics Essays 2, 76-100 (1989), p.91.

5. G. Feinberg, Phys.Rev. 159, 1089 (1967), - D17, 1651 (1978).

6. D. K. Deshpande, E.C.G. Sudarshan and O.M.P. Bilaniuk, Am.J.Phys. 30, 718 (1962)

7. S. Tanaka, Prog. Theor. Phys. (Kyoto) 24, 171 (1960).

8. L. H. Kauffman and H. P. Noyes, "Discrete Physics and the Derivation of Electromagnetism from the Formalism of Quantum Mechanics", Proc. Roy. Soc. A 452, 81-95 (1996).

9. D. O. McGoveran and H. P. Noyes, Physics Essays, 4, 115-120 (1991).

10. N. Bohr, Phil.Mag. 332, Feb. 1915.

11. H. P. Noyes, "Quantized Conic Sections; Quantum Gravity" in Proc. ANPA WEST 9 and SLAC-PUB-6057, March 15, 1993

12. H. P. Noyes, "Electromagnetism From Counting", in Physical Interpretations of Relativity Theory . III., M.C.Duffy, ed., Imperial College, September 1992 and SLAC-PUB-5858 (December, 1992); see also H.P.Noyes Proc. ANPA WEST 9 and SLAC-PUB-6057, p. 17.

13. H. P. Noyes DISCRETE PHYSICS: A New Fundamental Theory, J. C. van den Berg, ed. (in preparation).

14. A. C. Clarke, Interplanetary Flight, Temple Press, London, 1950.

15. P. S. Laplace, Le System du Monde, Paris, 1795; for quotation and discussion, see C. W. Misner, K. S. Thorne and J. A. Wheeler, Gravitation, W. H. Freeman and Co., 1973. 


\section{DISCLAMER}

Portions of this document may be illegible in electronic image products. Images are produced from the best available original document. 